\title{
THE FAILURE OF ANALYTICITY OF HAUSDORFF DIMENSIONS OF QUASI-CIRCLES OF FUCHSIAN GROUPS OF THE SECOND KIND
}

\author{
SHENGJIN HUO AND SHENGJIAN WU \\ (Communicated by Jeremy Tyson)
}

\begin{abstract}
Let $\Gamma$ be a Fuchsian group. Any $[\mu]$ in the Teichmüller space $T(\Gamma)$ determines a quasi-circle $f_{\mu}(\partial \mathbb{D})$. In this paper, we prove that, for any Fuchsian group $\Gamma$ of the second kind, the Hausdorff dimension $\delta([\mu])=\operatorname{dim} f_{\mu}(\partial \mathbb{D})$ is not a real analytic function in $T(\Gamma)$.
\end{abstract}

\section{INTRODUCTION AND MAIN RESULT}

A homeomorphism $f: \Omega \rightarrow \Omega^{\prime}$ between planar domains is called a $K$-quasiconformal mapping if it satisfies the Beltrami equation

$$
\bar{\partial} f=\mu \partial f, \text { a.e. } z \in \Omega,
$$

with a measurable coefficient $\mu$ with $\|\mu\|_{\infty} \leq k<1$, where $K=\frac{1+k}{1-k}$.

Let $\mathbb{D}$ be the unit disk in the complex plane $\mathbb{C}$ and $\partial \mathbb{D}$ be its boundary. Let $\Gamma$ be a Fuchsian group, i.e. a group of Möbius transformations acting properly discontinuously on $\mathbb{D}$. Then the accumulation set on $\partial \mathbb{D}$ of any orbit $\{g(z) ; g \in \Gamma\}$ of $z \in \mathbb{D}$ does not depend on $z$ and is called the limit set of $\Gamma$. We denote by $\Lambda(\Gamma)$ the limit set of $\Gamma$. If the limit set $\Lambda(\Gamma)$ agrees with $\partial \mathbb{D}, \Gamma$ is said to be of the first kind. Otherwise, we say that $\Gamma$ is of the second kind.

For a Fuchsian group $\Gamma$, we define

$$
M(\Gamma)=\left\{\mu \in L^{\infty}(\mathbb{D}):\|\mu\|_{\infty}<1 \text { and } \mu=\mu \circ g\left(\frac{\overline{g^{\prime}}}{g^{\prime}}\right), \forall g \in \Gamma\right\} .
$$

Let $\mu \in M(\Gamma)$. By the measurable Riemann mapping theorem, there exists a unique quasiconformal self-mapping $f^{\mu}$ of $\mathbb{D}$ fixing $1,-1$ and i, and satisfying

$$
\bar{\partial} f^{\mu}=\mu \partial f^{\mu} \text { a.e. } z \in \mathbb{D} \text {. }
$$

Similarly, there exists a unique quasiconformal homeomorphism $f_{\mu}$ of the plane $\mathbb{C}$ which is holomorphic outside the unit disk $\mathbb{D}$, fixing 1,-1 and $\mathrm{i}$, and satisfying

$$
\bar{\partial} f_{\mu}=\mu \partial f_{\mu} \text { a.e. } z \in \mathbb{D} \text {. }
$$

Received by the editors April 20, 2012 and, in revised form, March 11, 2013 and March 13, 2013.

2010 Mathematics Subject Classification. Primary 30C62, 30F35, 30F60.

Key words and phrases. Fuchsian group, Carleson measure, Hausdorff dimension, Teichmüller space.

The authors were supported by the National Natural Science Foundation of China (Grant No. 11371035 and Grant No. 11401432). 
Then $f_{\mu}(\partial \mathbb{D})$ is a quasi-circle, i.e. the image of $\partial \mathbb{D}$ under a quasiconformal mapping of the plane. If $\mu \in M(\Gamma)$, then $f^{\mu}$ has a well defined boundary value which is a quasi-symmetric homeomorphism of the unit circle. We define an equivalence relation on $M(\Gamma)$ by $\mu \sim \nu$ if

$$
\left.f^{\mu}\right|_{\partial \mathbb{D}}=\left.f^{\nu}\right|_{\partial \mathbb{D}} .
$$

The Teichmüller space $T(\Gamma)$ is by definition the quotient space $M(\Gamma) / \sim$. For more details about the properties of $T(\Gamma)$, we refer to $[8,9]$. Especially, for $[\mu] \in T(\Gamma)$, it is well known that $f_{\nu}(\partial \mathbb{D})=f_{\mu}(\partial \mathbb{D})$ for any $\nu \in[\mu]$.

Let $E \subset \mathbb{C}$ be a set. Now we recall the definition of Hausdorff dimension of $E$. For $0 \leq s \leq 2$ and $0<\delta \leq \infty$, we define

$$
\mathcal{H}_{\delta}^{s}(E)=\inf \left\{\sum_{i=1}^{\infty} \operatorname{diam}\left(B_{i}\right)^{s}: E \subset \bigcup_{i=1}^{\infty} B_{i}, \operatorname{diam}\left(B_{i}\right) \leq \delta\right\},
$$

where $B_{i} \subset \mathbb{C}$ is a set and $\operatorname{diam}\left(B_{i}\right)$ denotes its diameter, the infimum is taken over all open coverings of $E$. Then one defines the Hausdorff $s$-measure of $E$ to be

$$
\mathcal{H}^{s}(E)=\lim _{\delta \rightarrow 0} \mathcal{H}_{\delta}^{s}(E)=\sup _{\delta>0} \mathcal{H}_{\delta}^{s}(E) .
$$

The quantity $\mathcal{H}_{\infty}^{s}(E)$ is usually called the Hausdorff content of $E$. One defines the Hausdorff dimension of $E$ to be

$$
\operatorname{dim} E=\inf \left\{s: \mathcal{H}^{s}(E)=0\right\} .
$$

Now let $[\mu] \in T(\Gamma)$. Then we have a well defined map $\delta$ from $T(\Gamma)$ to $[1,2)$ which is denoted by $\delta([\mu])=\operatorname{dim} f_{\mu}(\partial \mathbb{D})$, i.e. the Hausdorff dimension of the quasi-circle $f_{\mu}(\partial \mathbb{D})$. It is known that if the Fuchsian group $\Gamma$ is finitely generated and of the first kind without parabolic element, the map $\delta: T(\Gamma) \rightarrow[1,2)$ is a real analytic function in $T(\Gamma)$. For more details about it, refer to [12. In [2, Astala and Zinsmeinster construct a Fuchsian group $\Gamma$ of the second kind such that the Hausdorff dimension $\delta([\mu])=\operatorname{dim}\left(f_{\mu}(\partial \mathbb{D})\right)$ is not real analytic in $T(\Gamma)$. In $[4$, they construct a holomorphically varying family of infinitely generated quasi-Fuchsian groups for which the Hausdorff dimension of the limit set is not real analytic. In [5], Bishop gave a criterion for failure of the analyticity for the Hausdorff dimension of the limit set of certain infinitely generated groups. It is easy to see that the Fuchsian groups studied in [4] and [5] are of the first kind.

In this paper, we shall prove the following result.

Theorem 1. For any Fuchsian group $\Gamma$ of the second kind, the map $\delta: T(\Gamma) \rightarrow$ $[1,2)$, given by $\delta([\mu])=\operatorname{dim} f_{\mu}(\partial \mathbb{D})$, is not a real analytic function in $T(\Gamma)$.

\section{PReliminaries AND LEMmaS}

In order to prove Theorem 1, we first recall some basic definitions and results related to the theory of quasiconformal mappings and harmonic analysis. For more detail, the reader can refer to [7], 8].

Let $Q S(\partial \mathbb{D})$ denote the set of all quasi-symmetric homeomorphism of $\partial \mathbb{D}$. A homeomorphism of the unit circle is called strongly quasi-symmetric if it is absolutely continuous at every scale, i.e., if for any $\varepsilon>0$, there exists $\delta>0$, for any interval $I$ and any Borel set $E \subset I$, such that

$$
|E| \leq \delta|I| \Longrightarrow|h(E)| \leq \varepsilon|h(I)| .
$$


We denote by $S Q S(\partial \mathbb{D})$ the set of strongly quasi-symmetric homeomorphisms of $\partial \mathbb{D}$. Naturally, a strongly quasi-symmetric homeomorphism is quasi-symmetric. But the converse is far from being true, since a quasi-symmetry may be singular. For a Fuchsian group $\Gamma$, we define by $Q S(\Gamma)$ the set of quasi-symmetric homeomorphisms $h \in Q S(\partial \mathbb{D})$ such that, for all $\gamma \in \Gamma,\left.h \circ \gamma \circ h^{-1}\right|_{\partial \mathbb{D}}=\left.\theta(\gamma)\right|_{\partial \mathbb{D}}$, where $\theta(\gamma)$ is a Möbius transformation.

One of the main tools in the proof of Theorem 1 is the Carleson measures. Let $\xi \in \mathbb{C}$ and $R>0$ be a real number. In this paper, we shall use $B(\xi, R)$ to denote the disk centered at $\xi$ with radius $R$. A positive measure $m$ in a domain $\Omega$ of the complex plane is called a Carleson measure if there exists a constant $C$ such that

$$
m(B(\xi, R) \cap \Omega) \leq C R,
$$

for all $\xi \in \partial \Omega$ and $0<R \leq \operatorname{diam} \Omega$. The smallest constant $C$ which satisfies the above inequality is called the Carleson norm of $m$.

In this paper, we denote by $C M(\mathbb{D})$ the set of measurable functions $\mu$ in the unit disk such that

$$
\frac{|\mu|^{2}}{1-|z|^{2}} d x d y
$$

is a Carleson measure.

In the proof of Theorem 1, we need the following lemma.

Lemma 2.1 ([7, [13]). Let $\Omega$ be a Lavrentiev domain, i.e. a bilipschitz image of the unit disk. Then the following are equivalent:

(a) $d \nu$ is a Carleson measure for $\Omega$.

(b) For $0<p<\infty$ and $f \in H^{p}(\Omega)$,

$$
\iint_{\Omega}|f|^{p} d \nu \leq C \int_{\partial \Omega}|f|^{p} d s
$$

where $H^{p}(\Omega)=\left\{f: f\right.$ is analytic on $\Omega$ and $\left.\int_{\partial \Omega}|f|^{p} d s<\infty\right\}$, and the constant $C$ depends only on the Carleson norm of $d \nu$.

Remark. The above lemma was first given by Carleson [7, Theorem 3.9, p. 61] when $\Omega$ is the upper half plane. Zinsmeister proved that Carleson's theorem remains true for Lavrentiev domains; see [13.

\section{Proof of Theorem 1}

Let $\Gamma$ be a Fuchsian group of the second kind. In order to prove the theorem, we shall construct a $\mu \in M(\Gamma) \cap C M(\mathbb{D})$ such that $\operatorname{dim}_{\mu}(\partial \mathbb{D})>1$. Note that the existence of $\mu^{*} \in C M(\mathbb{D})$ such that $\operatorname{dim}_{\mu^{*}}(\partial \mathbb{D})>1$ can be found in $([3]$, p. 624).

Now let $P$ be a fundamental domain of $\Gamma$; see Figure 1 .

Since $\Gamma$ is of the second kind, we can take a free side $\alpha \subset \bar{P} \cap \partial \mathbb{D}$ of $P$. Divide $\alpha$ into three disjoint (except the endpoints) arcs $\alpha_{1}, \alpha_{2}$ and $\alpha_{3}$ in counterclockwise order with equal length; see Figure 1 . We choose a disk $B_{0}$ such that $B_{0} \cap \mathbb{D}$ is a nonempty subset of $P$ and $B_{0} \cap \partial \mathbb{D}=\alpha_{2}$. Set $B_{P}=B_{0} \cap \mathbb{D}$. For some technical reasons, we also need the inner angle in $B_{P}$ between $\partial B_{0}$ and $\partial \mathbb{D}$ to be less than $\frac{\pi}{4}$. We can use a smooth curve to connect two sides of $P$ and divide $P$ into two parts such that the component which contains $B_{P}$ is a simply connected Lavrentiev domain, which is denoted by $P^{*}$; see Figure 1 . 


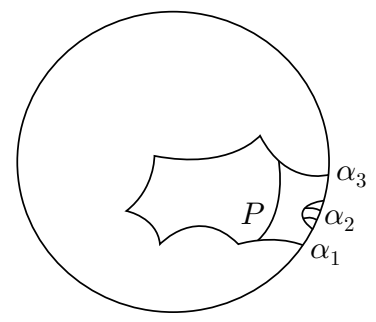

FIGURE 1

Let $\mu^{*} \in C M(\mathbb{D})$ be a measurable function in the unit disk with $\left\|\mu^{*}\right\|_{\infty}<1$ such that $\operatorname{dim} f_{\mu^{*}}(\partial \mathbb{D})>1$. Then we can find a small arc $\alpha_{0}$ of $\partial \mathbb{D}$ such that $\operatorname{dim} f_{\mu^{*}}\left(\alpha_{0}\right)>1$. Without loss of generality, we may assume that $\alpha_{0}=\alpha_{2}$ and $f^{\mu^{*}}\left(\alpha_{2}\right)=\alpha_{2}$. Otherwise, we can conjugate $f^{\mu^{*}}$ by two Möbius transformations $g_{1}^{*}, g_{2}^{*} \in A U T(\mathbb{D})$, where $A U T(\mathbb{D})$ denotes the biholomorphic automorphism group of the unit disk $\mathbb{D}$, such that the composition $g_{1}^{*} \circ f^{\mu^{*}} \circ g_{2}^{*-1}$ satisfies the conditions that we need.

Let

$$
\mu_{P}(z)=\left\{\begin{array}{c}
\mu^{*}(z), \quad z \in B_{P} \\
0, \quad z \in P-B_{P} .
\end{array}\right.
$$

Now we translate $\mu_{P}$ by the group $\Gamma$ to the whole disk $\mathbb{D}$. Then we get a measurable function $\mu \in M(\Gamma)$. We shall prove that $\mu \in C M(\mathbb{D})$. We first prove a property of $\mu_{P}$.

Claim. There exists a constant $C_{0}$ which depends only on the Carleson norm of $\mu^{*}$ such that for any $x \in \bar{P}^{*}$, and $0<r \leq \operatorname{diam} P^{*}$,

$$
\iint_{B(x, r) \cap \overline{P^{*}}} \frac{\left|\mu_{P}\right|^{2}}{1-|z|^{2}} d x d y \leq C_{0} r,
$$

where $\bar{P}^{*}$ denotes the closure of $P^{*}$ in $\mathbb{C}$.

In order to prove the claim, it is easy to see that, since $\mu_{P}$ is supported on $\overline{B_{P}}$, we need only to treat the case that $x \in \overline{B_{P}}$. Let $C^{*}$ be the Carleson norm of $\mu^{*}$. If $r \geq \operatorname{diam} B_{P}$, then it is easy to see that $B_{P} \subset B(\xi, r) \cap \mathbb{D}$, where $\xi$ is the middle point of $\alpha_{2}$. Note that $\frac{\left|\mu^{*}\right|^{2}}{1-|z|^{2}}$ is a Carleson measure of $\mathbb{D}$. Thus we have

$$
\iint_{B(x, r) \cap P^{*}} \frac{\left|\mu_{P}\right|^{2}}{1-|z|^{2}} d x d y \leq \iint_{B_{P}} \frac{\left|\mu_{P}\right|^{2}}{1-|z|^{2}} d x d y \leq C^{*} r .
$$

Now suppose $0<r<\operatorname{diam} B_{P}$. We need to study two cases. First we assume that $\operatorname{dist}(x, \partial \mathbb{D}) \geq 2 r$ (obviously $r<1$ ), where $\operatorname{dist}(\cdot, \cdot)$ denotes the Euclidean distance. Then in this case we have

$$
\iint_{B(x, r) \cap P^{*}} \frac{\left|\mu_{P}\right|^{2}}{1-|z|^{2}} d x d y \leq \frac{\pi r^{2}}{1-(1-r)^{2}}=\frac{\pi r}{2-r} \leq \pi r .
$$




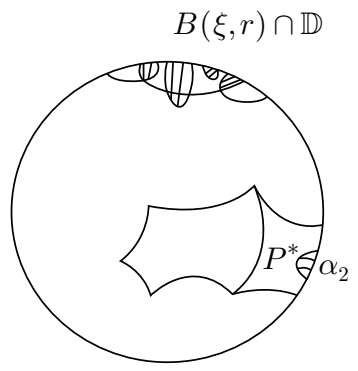

FiguRE 2

Next we assume that $\operatorname{dist}(x, \partial \mathbb{D})<2 r$. In this case, we can choose a point $\eta \in \partial \mathbb{D}$ such that $\operatorname{dist}(\eta, x)<2 r$. Therefore we have

$$
\iint_{B(x, r) \cap P^{*}} \frac{\left|\mu_{P}\right|^{2}}{1-|z|^{2}} d x d y \leq \iint_{B(\eta, 4 r) \cap P^{*}} \frac{\left|\mu_{P}\right|^{2}}{1-|z|^{2}} d x d y \leq 4 C^{*} r .
$$

By setting $C_{0}=\max \left\{\pi, 4 C^{*}\right\}$, we see that $C_{0}$ satisfies the conclusion of the claim.

Now we are able to prove that $\mu \in C M(\mathbb{D})$. Let $\xi$ be an arbitrary point of $\partial \mathbb{D}$ and let $r$ be a positive real number; see Figure 2. We divide the proof into the following two cases.

Case 1. There exists $g \in \Gamma$ such that $g^{-1}(B(\xi, r) \cap \mathbb{D}) \subset P$. It is easy to see that the length of the boundary of $B(\xi, r) \cap \mathbb{D}$ is less than $2 \pi r$ and $g^{-1}(B(\xi, r) \cap \mathbb{D})$ is a Lavrentiev domain in $P$. Note that $\mu$ is $g$ invariant and $g \in A U T(\mathbb{D})$. It follows from the claim that the restriction of $\nu=\frac{|\mu|^{2}}{1-|z|^{2}} d x d y$ to $g^{-1}(B(\xi, r) \cap \mathbb{D})$ is a Carleson measure for the domain $g^{-1}(B(\xi, r) \cap \mathbb{D})$ and the Carleson norm is less than or equal to $C_{0}$. Therefore, by Lemma 2.1 we deduce that there exists a constant $C_{1}$ which depends only on $C_{0}$ such that

$$
\begin{aligned}
\iint_{B(\xi, r) \cap \mathbb{D}} \frac{|\mu(w)|^{2}}{1-|w|^{2}} d u d v & =\iint_{g^{-1}(B(\xi, r) \cap \mathbb{D})} \frac{|\mu(g(z))|^{2}}{1-|g(z)|^{2}}\left|g^{\prime}(z)\right|^{2} d x d y \\
& =\iint_{g^{-1}(B(\xi, r) \cap \mathbb{D})} \frac{\left|\mu(g(z)) \frac{\overline{g^{\prime}(z)}}{g^{\prime}(z)}\right|^{2}}{1-|g(z)|^{2}}\left|g^{\prime}(z)\right|^{2} d x d y \\
& =\iint_{g^{-1}(B(\xi, r) \cap \mathbb{D})} \frac{|\mu(z)|^{2}}{1-|z|^{2}}\left|g^{\prime}(z)\right| d x d y \\
& \leq C_{1} \int_{\partial\left(g^{-1}(B(\xi, r) \cap \mathbb{D})\right)}\left|g^{\prime}(z)\right| d s \\
& =C_{1} \int_{\partial(B(\xi, r) \cap \mathbb{D})} d s \leq 2 \pi C_{1} r .
\end{aligned}
$$

Case 2. There exists more than one $g \in \Gamma$ such that $g\left(B_{P}\right) \cap(B(\xi, r) \cap \mathbb{D}) \neq$ $\emptyset$. In this case, we denote by $\Gamma^{\prime}$ the set of the Möbius maps $g \in \Gamma$ such that $g\left(B_{P}\right) \cap(B(\xi, r) \cap \mathbb{D}) \neq \emptyset$. Since $\Gamma$ is a Fuchsian group, there are at most countable elements in $\Gamma^{\prime}$. 
Let $g \in \Gamma^{\prime}$. We first suppose that $g\left(B_{P}\right) \subset B(\xi, r) \cap \mathbb{D}$; see Figure 2. By using the same method as we used in the proof of Case 1 , we have

$$
\begin{aligned}
\nu\left(g\left(B_{P}\right)\right) & =\iint_{g\left(B_{P}\right)} \frac{|\mu(w)|^{2}}{1-|w|^{2}} d u d v \\
& =\iint_{B_{P}} \frac{\left|\mu(g(z)) \frac{\overline{g^{\prime}(z)}}{g^{\prime}(z)}\right|^{2}}{1-|g(z)|^{2}}\left|g^{\prime}(z)\right|^{2} d x d y \\
& =\iint_{B_{P}} \frac{|\mu(z)|^{2}}{1-|z|^{2}}\left|g^{\prime}(z)\right| d x d y \\
& \leq C_{1} \int_{\partial B_{P}}\left|g^{\prime}(z)\right| d s \\
& \leq \pi C_{1} L\left(g\left(\alpha_{2}\right)\right),
\end{aligned}
$$

where $L\left(g\left(\alpha_{2}\right)\right)$ denote the length of $g\left(\alpha_{2}\right)$.

We next suppose that $g\left(B_{P}\right)$ is not contained in $B(\xi, r) \cap \mathbb{D}$. Then there are at most two possibilities.

One possibility is that $\partial g\left(B_{P}\right) \cap \partial \mathbb{D} \subset B(\xi, r) \cap \partial \mathbb{D}$; see Figure 2. It is easy to see that

$$
\nu\left(g\left(B_{P}\right) \cap B(\xi, r)\right) \leq \nu\left(g\left(B_{P}\right)\right) \leq \pi C_{1} L\left(g\left(\alpha_{2}\right)\right) .
$$

Another possibility is that $\partial g\left(B_{P}\right) \cap \partial \mathbb{D}$ is not contained in $B(\xi, r) \cap \partial \mathbb{D}$. In this case, we see that $g\left(B_{P}\right) \cap B(\xi, r)$ is a spherical triangle, i.e. a triangle whose sides are sub-arcs of some circles and the sum of the interior angles is larger than $\pi$; see Figure 2. Note that one inner angle between $\partial \mathbb{D}$ and $\partial B_{0}$ is less than $\frac{\pi}{4}$ and another inner angle between $\partial \mathbb{D}$ and $\partial B(\xi, r)$ is at most $\frac{\pi}{2}$. By a simple calculation, we can see that the length of the perimeter of the spherical triangle $g\left(B_{P}\right) \cap B(\xi, r) \cap \mathbb{D}$ is less than $4 \pi$ times the length of the side $g\left(\alpha_{2}\right) \cap(B(\xi, r) \cap \partial \mathbb{D})$. We deduce that

$\nu\left(g\left(B_{P}\right) \cap B(\xi, r) \cap \mathbb{D}\right) \leq C_{1} L\left(\partial\left(g\left(B_{P}\right) \cap B(\xi, r) \cap \mathbb{D}\right)\right) \leq 4 \pi C_{1} L\left(g\left(\alpha_{2}\right) \cap(B(\xi, r) \cap \partial \mathbb{D})\right)$.

Therefore, by using the above estimates, we conclude that

$$
\nu(\mathbb{D} \cap B(\xi, r)) \leq \sum_{g \in \Gamma^{\prime}} \nu\left(g\left(B_{P}\right)\right) \leq 4 \pi C_{1} L(\partial \mathbb{D} \cap B(\xi, r)) .
$$

We have proved

$$
\nu(B(\xi, r) \cap \mathbb{D}) \leq C_{2} r,
$$

where $C_{2}=4(\pi)^{2} C_{1}$. Hence $\mu \in C M(\mathbb{D})$. Furthermore, we know from ([6], Theorem 1) that $\left.f^{\mu}\right|_{\partial \mathbb{D}} \in S Q S(\partial \mathbb{D})$.

Now we prove $\operatorname{dimf}_{\mu}(\partial \mathbb{D})>1$. In fact, if we set $\mu(z)=\mu^{*}(z) \equiv 0,\left(z \in B_{0} \backslash B_{p}\right)$, then $\mu(z)$ and $\mu^{*}(z)$ have the same value in the ball $B_{0}$. Thus $f_{\mu} \circ f_{\mu^{*}}^{-1}$ is conformal in $f_{\mu^{*}}\left(B_{0}\right)$. Noting that conformal maps preserve the Hausdorff dimension, we have

$$
\operatorname{dim} f_{\mu}\left(\alpha_{2}\right)=\operatorname{dim} f_{\mu} \circ f_{\mu^{*}}^{-1} \circ f_{\mu^{*}}\left(\alpha_{2}\right)=\operatorname{dim} f_{\mu^{*}}\left(\alpha_{2}\right)>1 .
$$


Finally, we prove that $\operatorname{dim}\left(f_{t \mu}(\partial \mathbb{D})\right)$ is not real analytic with respect to $t \in \mathbb{D}$. The proof of this fact is the same as Astala and Zinsmeister gave in ([3]). For the reader's convenience, we give the details here. Consider the mapping $\pi: t \rightarrow\left[f^{t \mu}\right]$, where $t \in \overline{\mathbb{D}}$ and $f^{t \mu}$ is the self-mapping of the unit disk which has the complex dilatation $t \mu$ in $\mathbb{D}$. As is well known, $\pi$ is a holomorphic mapping from $\overline{\mathbb{D}}$ to $T(\Gamma)$. Therefore it suffices to show that $\delta \circ \pi$ is not real analytic on $\mathbb{D}$. Since $\mu \in C M(\mathbb{D})$, from [10], we know that there is a constant $\varepsilon>0$, such that, for all $|t|<\varepsilon, f_{t \mu}(\mathbb{D})$ is a Lavrentiev domain. In particular, $\delta \circ \pi$ is equal to 1 in a neighborhood of 0 . This implies that $\delta \circ \pi$ cannot be real analytic in $\mathbb{D}$, since $\delta \circ \pi(1)>1$. The proof of Theorem 1 is completed.

Remark. A Fuchsian group $\Gamma$ is of divergence type if $\sum_{\gamma \in \Gamma}(1-|\gamma(0)|)=+\infty$. Otherwise, it is called convergence type. It is well known that all divergence type group is of the first kind. Agard and Pommerenke ([1, [11]) showed that if $\Gamma$ is of divergence type and if $h \in Q S(\Gamma)$, then $h$ must be singular. On the other hand, Astala and Zinsmeister ([2]) showed that if $\Gamma$ is of convergence type, there always exists $\mu \in C M(\mathbb{D}) \cap M(\Gamma)$, such that $\left.f^{\mu}\right|_{\partial \mathbb{D}} \in S Q S(\mathbb{D}) \cap Q S(\Gamma)$. From the proof of Theorem 1, we can see that, for a Fuchsian group of the second kind, there exists not only $\left.f^{\mu}\right|_{\partial \mathbb{D}} \in S Q S(\mathbb{D}) \cap Q S(\Gamma)$, but also $\operatorname{dim} f^{\mu}(\partial \mathbb{D})>1$. It is natural to ask whether there exists $\mu \in C M(\mathbb{D}) \cap M(\Gamma)$ such that $\operatorname{dim} f^{\mu}(\partial \mathbb{D})>1$ for all convergence type group $\Gamma$.

\section{REFERENCES}

[1] Stephen Agard, A geometric proof of Mostow's rigidity theorem for groups of divergence type, Acta Math. 151 (1983), no. 3-4, 231-252, DOI 10.1007/BF02393208. MR723011(86b:22017)

[2] Kari Astala and Michel Zinsmeister, Mostow rigidity and Fuchsian groups (English, with French summary), C. R. Acad. Sci. Paris Sér. I Math. 311 (1990), no. 6, 301-306. MR.1071631 (92c:30021)

[3] Kari Astala and Michel Zinsmeister, Teichmüller spaces and BMOA, Math. Ann. 289 (1991), no. 4, 613-625, DOI 10.1007/BF01446592. MR1103039 (92k:30031)

[4] K. Astala and M. Zinsmeister, Holomorphic families of quasi-Fuchsian groups, Ergodic Theory Dynam. Systems 14 (1994), no. 2, 207-212, DOI 10.1017/S0143385700007847. MR:1279468 (95k:30095)

[5] Christopher J. Bishop, A criterion for the failure of Ruelle's property, Ergodic Theory Dynam. Systems 26 (2006), no. 6, 1733-1748, DOI 10.1017/S014338570600071X. MR2279263 (2008j:37097)

[6] Guizhen Cui and Michel Zinsmeister, BMO-Teichmüller spaces, Illinois J. Math. 48 (2004), no. 4, 1223-1233. MR2114154 (2005h:30038)

[7] John B. Garnett, Bounded analytic functions, Pure and Applied Mathematics, vol. 96, Academic Press Inc. [Harcourt Brace Jovanovich Publishers], New York, 1981. MR628971 (83g:30037)

[8] Olli Lehto, Univalent functions and Teichmüller spaces, Graduate Texts in Mathematics, vol. 109, Springer-Verlag, New York, 1987. MR867407 (88f:30073)

[9] Subhashis Nag, The complex analytic theory of Teichmüller spaces, Canadian Mathematical Society Series of Monographs and Advanced Texts, John Wiley \& Sons Inc., New York, 1988. A Wiley-Interscience Publication. MR927291 (89f:32040)

[10] Ch. Pommerenke, Schlichte Funktionen und analytische Funktionen von beschränkter mittlerer Oszillation (German), Comment. Math. Helv. 52 (1977), no. 4, 591-602. MR0454017 (56 \#12268)

[11] Ch. Pommerenke, Polymorphic functions for groups of divergence type, Math. Ann. 258 (1981/82), no. 4, 353-366, DOI 10.1007/BF01453971. MR650942 (83c:30048) 
[12] David Ruelle, Repellers for real analytic maps, Ergodic Theory Dynamical Systems 2 (1982), no. 1, 99-107. MR684247 (84f:58095)

[13] Michel Zinsmeister, Les domaines de Carleson (French), Michigan Math. J. 36 (1989), no. 2, 213-220, DOI 10.1307/mmj/1029003944. MR.1000525 (90i:30054)

LMam and School of Mathematical Sciences, Peking University, Beijing, 100871, People's Republic of China

E-mail address: sjhuo@pku.edu.cn

Current address: Department of Mathematics, Tianjin Polytechnic University, Tianjin, 300387, People's Republic of China

LMam and School of Mathematical Sciences, Peking University, Beijing, 100871, People's Republic of China

E-mail address: wusj@math.pku.edu.cn 